\title{
CERTAIN OPERATORS AND FOURIER TRANSFORMS ON $L^{2}$
}

\author{
RICHARD R. GOLDBERG
}

1. Introduction. A well known theorem of Titchmarsh [2] states that if $f \in L^{2}(0, \infty)$ and if $g$ is the Fourier cosine transform of $f$, then $G(x)=x^{-1} \int_{0}^{x} g(y) d y$ is the cosine transform of $F(y)=\int_{\nu}^{\infty}(f(x) / x) d x$ (both $F$ and $G$ being in $L^{2}$ ). The same result applies to sine transforms.

In this paper we prove the following result for a wide class of functions $\psi$ : If $g$ is the cosine transform of $f \in L^{2}$ then

$$
G(x)=x^{-1} \int_{0}^{\infty} \psi(y / x) g(y) d y
$$

is the cosine transform of $F(y)=\int_{0}^{\infty} x^{-1} \psi(y / x) f(x) d x$. (The same result again applies to sine transforms.) The theorem of Titchmarsh stated above is the special case of our result in which $\psi$ is the characteristic function of $(0,1)$.

We shall prove the above result by developing properties of a certain class of bounded operators on $L^{2}$.

Finally we shall construct a class of self-adjoint bounded operators which commute with the Fourier cosine (or sine) transform.

2. Preliminaries. We shall denote $L^{p}(0, \infty)$ by $L^{p},(p=1,2)$ with the $L^{p}$ norm $\|f\|_{p}$ defined as usual as $\left(\int_{0}^{\infty}|f(x)|^{p} d x\right)^{1 / p}$. If $T$ is a linear transformation on $L^{2}$ into itself then $\|T\|$ is defined as

$$
\operatorname{lub}_{0 \in L^{2}}\|T g\|_{2} /\|g\|_{2} \text {. }
$$

We shall make use of the

Schwarz Inequality: if $f, g \in L^{2}$ then $f g \in L^{\prime}$ and $\|f g\|_{1} \leqq\|f\|_{2}\|g\|_{2}$, and its

Converse: if for each $h \in L^{2},\|G h\|_{1} \leqq A\|h\|_{2}$ then $G \in L^{2}$ and $\|G\|_{2} \leqq A$.

3. A Class of bounded operators on $L^{2}$.

LemmA. If $\psi(y) \geqq 0$ and $\int_{0}^{\infty} \psi(y) y^{-1 / 2} d y=A<\infty$ then for any $g, h \in L^{2}$

$$
\int_{0}^{\infty} \psi(y) d y \int_{0}^{\infty}|h(x) g(x y)| d x \leqq A\|h\|_{2}\left\|_{g}\right\|_{2} .
$$

Proof. For $y>0$

Received by the editors October 30, 1958. 


$$
\int_{0}^{\infty}|g(x y)|^{2} d x=\frac{1}{y} \int_{0}^{\infty}|g(x)|^{2} d x .
$$

Therefore, by the Schwarz Inequality,

$$
\int_{0}^{\infty}|h(x) g(x y)| d x \leqq\|h\|_{2} \cdot \frac{1}{y^{1 / 2}}\|g\|_{2} .
$$

Hence

$$
\int_{0}^{\infty} \psi(y) d y \int_{0}^{\infty}|h(x) g(x y)| d x \leqq\|h\|_{2}\|g\|_{2} \int_{0}^{\infty} \psi(y) y^{-1 / 2} d y=A\|h\|_{2}\|g\|_{2} .
$$

The first part of the next theorem was proved in a much different form by Schur [1].

Theorem 1. Let $\psi$ be non-negative with $\int_{0}^{\infty} \psi(y) y^{-1 / 2} d y=A<\infty$. Let $\psi$ define the linear transformation $T$ on $L^{2}$ as follows:

$$
T g=G \text { means } G(x)=\frac{1}{x} \int_{0}^{\infty} \psi\left(\frac{y}{x}\right) g(y) d y \quad\left(g \in L^{2}\right) .
$$

Then $T$ is a bounded operator on $L^{2}$ and $\|T\| \leqq A$.

Furthermore if we define $T^{*}$ as

$$
T^{*} f=F \text { means } F(x)=\int_{0}^{\infty} \frac{1}{y} \psi\left(\frac{x}{y}\right) f(y) d y \quad\left(f \in L^{2}\right),
$$

then $T^{*}$ is the adjoint of $T$ and so $\left\|T^{*}\right\| \leqq A$.

Proof. We shall first show that $G \in L^{2}$ and that $\|G\|_{2} \leqq A\|g\|_{2}$.

For any $h \in L^{2}$ we have

$$
\begin{aligned}
& \int_{0}^{\infty}|G(x) h(x)| d x \leqq \int_{0}^{\infty} \frac{|h(x)|}{x} d x \int_{0}^{\infty} \psi\left(\frac{y}{x}\right)|g(y)| d y \\
& =\int_{0}^{\infty}|h(x)| d x \int_{0}^{\infty} \psi(y)|g(x y)| d y=\int_{0}^{\infty} \psi(y) d y \int_{0}^{\infty}|h(x) g(x y)| d x .
\end{aligned}
$$

The last iterated integral converges (absolutely) by the lemma justifying the change in order of integration. Thus by the lemma

$$
\|G h\|_{1} \leqq A\|g\|_{2}\|h\|_{2} \text {. }
$$

The converse of the Schwarz Inequality thus implies that

$$
G \in L^{2} \text { and }\|G\|_{2} \leqq A\|g\|_{2} .
$$

Since $G=T g$ this shows that $\|T g\|_{2} \leqq A\|g\|_{2}$ for all $g \in L^{2}$ and so $T$ is a 
bounded linear transformation on $L^{2}$ into itself (bounded operator) and $\|T\| \leqq A$. The first part of the theorem is thus established.

Now choose any $f, g \in L^{2}$. Then with $(a, b)$ defined as $\int_{0}^{\infty} a(x) b(x) d x$, the usual inner product in $L^{2}$, we have

$$
(T g, f)=\int_{0}^{\infty} \frac{f(x)}{x} d x \int_{0}^{\infty} \psi\left(\frac{y}{x}\right) g(y) d y,
$$

and

$$
\left(g, T^{*} f\right)=\int_{0}^{\infty} g(y) d y \int_{0}^{\infty} \frac{1}{x} \psi\left(\frac{y}{x}\right) f(x) d x .
$$

The integrals in (1) and (2) converge absolutely by the lemma and hence are equal. Thus

$$
(T g, f)=\left(g, T^{*} f\right)
$$

which, by definition of adjoint, shows that $T^{*}$ is the adjoint of $T$. Finally, since $\left\|T^{*}\right\|=\|T\|$, we have $\left\|T^{*}\right\| \leqq A$ and the proof is complete.

In passing we remark that the integrals defining $F$ and $G$ in the statement of Theorem 1 exist only almost everywhere.

4. Relation to Fourier transforms. We shall write $U f=g$ if $g$ is the Fourier cosine transform of $f$. Thus if $U f=g$ then

$$
g(y)=\underset{R \rightarrow \infty}{\operatorname{lix.m}}\left(\frac{2}{\pi}\right)^{1 / 2} \int_{0}^{R} f(t) \cos y t d t \quad f \in L^{2},
$$

where 1.i.m. stands for limit in the $L^{2}$ mean. Furthermore

$$
g(y)=\left(\frac{2}{\pi}\right)^{1 / 2} \int_{0}^{\infty} f(t) \cos y t d t \quad \text { if } f \in L^{\prime} \cap L^{2},
$$

the above holding for almost all $y$.

It is well known that if $f \in L^{2}$ and $U f=g$ then $g \in L^{2}$ and $U g=f$. Moreover $U$ is a self-adjoint operator $\left(U=U^{*}\right)$.

It will be readily verified that everything we prove about the Fourier cosine transform $U$ will also hold for the Fourier sine transform.

TheOREM 2. If $\psi$ is non-negative, $\psi \in L^{\prime}$, and $\int_{0}^{\infty} \psi(y) y^{-1 / 2} d y<\infty$ then

$$
T U=U T^{*}
$$

where $T, T^{*}$ are as in Theorem 1.

Proof. It is sufficient to prove 


$$
T U f=U T^{*} f \text { for } f \in L^{\prime} \cap L^{2}
$$

since $L^{\prime} \cap L^{2}$ is dense in $L^{2}$ and $T, T^{*}, U$ are continuous on $L^{2}$.

Accordingly, choose any $f \in L^{\prime} \cap L^{2}$ and let

$$
g=U f, \quad G=T g, \quad F=T^{*} f .
$$

We need only show that $G=U F$. With $c=(2 / \pi)^{1 / 2}$ we have

$$
\begin{aligned}
G(x) & =\frac{1}{x} \int_{0}^{\infty} \psi\left(\frac{y}{x}\right) g(y) d y=\frac{c}{x} \int_{0}^{\infty} \psi\left(\frac{y}{x}\right) d y \int_{0}^{\infty} f(t) \cos y t d t \\
& =\frac{c}{x} \int_{0}^{\infty} f(t) d t \int_{0}^{\infty} \psi\left(\frac{y}{x}\right) \cos y t d y \\
& =c \int_{0}^{\infty} f(t) d t \int_{0}^{\infty} \psi(y) \cos x y t d y \\
& =c \int_{0}^{\infty} \frac{f(t)}{t} d t \int_{0}^{\infty} \psi\left(\frac{y}{t}\right) \cos x y d y \\
& =c \int_{0}^{\infty} \cos x y d y \int_{0}^{\infty} \frac{1}{t} \psi\left(\frac{y}{t}\right) f(t) d t \\
& =c \int_{0}^{\infty} F(y) \cos x y d y .
\end{aligned}
$$

The integral in (3) converges absolutely since $\psi, f \in L^{\prime}$. This justifies the changes in order of integration and also shows that $F \in L^{\prime}$. Thus $G=U F$ which is what we wished to show.

REMARK. If we set

$$
\begin{array}{ll}
\psi(y)=1, & 0 \leqq y \leqq 1 ; \\
\psi(y)=0, & y>1,
\end{array}
$$

then if $G=T g, F=T^{*} f$ we have

$$
G(x)=\frac{1}{x} \int_{0}^{x} g(y) d y, \quad F(y)=\int_{y}^{\infty} \frac{f(x)}{x} d x .
$$

From Theorem 2 we see that if $g=U f$ then $G=U F$. This is the theorem of Titchmarsh mentioned in the introduction.

5. A more general result. We may drop the hypothesis that $\psi \in L^{\prime}$ in Theorem 2. To see this choose any non-negative $\psi$ such that $\int_{0}^{\infty} \psi(y) y^{-1 / 2} d y=A<\infty$ (but not necessarily such that $\psi \in L^{\prime}$ ). For $n=1,2, \cdots$ define 


$$
\begin{array}{ll}
\psi_{n}(y)=\psi(y), & 1 / n \leqq y \leqq n ; \\
\psi_{n}(y)=0, & 0 \leqq y<1 / n ; n<y<\infty .
\end{array}
$$

Then $\int_{0}^{\infty} \psi_{n}(y) y^{-1 / 2} d y=A_{n}<\infty$ and, by the Lebesgue convergence theorem,

$$
\lim _{n \rightarrow \infty} A_{n}=A .
$$

Moreover if $T, T_{n}$ are defined by $\psi, \psi_{n}$ as in Theorem 1 then $T-T_{n}$ is defined by $\psi-\psi_{n}$ and thus, by Theorem 1 ,

$$
\left\|T-T_{n}\right\| \leqq A-A_{n} \rightarrow 0 \quad \text { as } n \rightarrow \infty .
$$

But $\psi_{n}$ obeys the hypotheses of Theorem 2 . Hence

$$
T_{n} U=U T_{n}^{*} .
$$

Letting $n \rightarrow \infty$ and using (4) we have

$$
T U=U T^{*} .
$$

We have thus shown that $T U=U T^{*}$ even for $T$ defined by a nonnegative $\psi$ for which we assume only $\int_{0}^{\infty} \psi(y) y^{-1 / 2} d y<\infty$. We now state this in detail.

Theorem 3. Let $\psi$ be non-negative with $\int_{0}^{\infty} \psi(y) y^{-1 / 2} d y<\infty$. Define the linear transformation $T$ on $L^{2}$ as follows:

$$
T g=G \text { means } G(x)=\frac{1}{x} \int_{0}^{\infty} \psi\left(\frac{y}{x}\right) g(y) d y .
$$

Then $T$ is a bounded operator on $L^{2}$. Moreover if $T^{*}$ is the adjoint of $T$ and $U$ is the Fourier cosine transform then

$$
T U=U T^{*} .
$$

REMARK. This theorem, translated back into classical terminology, is the generalization of the theorem of Titchmarsh stated in the introduction.

6. Operators that commute with the cosine transform. In order for $T$ to be self-adjoint $\left(T=T^{*}\right)$ we see from the definition of $T, T^{*}$ in Theorem 1 that it is sufficient to have

$$
\frac{1}{x} \psi\left(\frac{y}{x}\right)=\frac{1}{y} \psi\left(\frac{x}{y}\right), \quad 0<x, y<\infty ;
$$

or 


$$
\psi(y)=\frac{1}{y} \psi\left(\frac{1}{y}\right), \quad 0<y<\infty .
$$

Suppose then that we have a non-negative function $\psi$ defined on $(0,1]$ such that

$$
\int_{0}^{1} \psi(y) y^{-1 / 2} d y<\infty
$$

and define $\psi(y)$ for $y>1$ by

$$
\psi(y)=\frac{1}{y} \psi\left(\frac{1}{y}\right)
$$

Then if $y_{1}<1$ we have

$$
\psi\left(\frac{1}{y_{1}}\right)=y_{1} \psi\left(y_{1}\right)
$$

so that $\psi(y)=(1 / y) \psi(1 / y)$ for all $y>0$ (i.e. (5) holds). From (5) and (6) we have

$$
\int_{1}^{\infty} \psi(y) y^{-1 / 2} d y=\int_{1}^{\infty} \psi\left(\frac{1}{y}\right) y^{-3 / 2} d y=\int_{0}^{1} \psi(y) y^{-1 / 2} d y<\infty .
$$

This and (6) imply

$$
\int_{0}^{\infty} \psi(y) y^{-1 / 2} d y<\infty
$$

so that the hypotheses of Theorem 3 hold. From (5) we conclude that the $T$ defined by $\psi$ is self-adjoint so that we have the following consequence of Theorem 3 .

Theorem 4. Let $\psi$ be non-negative on $(0,1]$ with $\int_{0}^{1} \psi(y) y^{-1 / 2} d y<\infty$. Define $\psi(y)=(1 / y) \psi(1 / y)$ for $y>1$. Then if $T$ is as in Theorem 1

$$
T U=U T \text {. }
$$

In other words $T$ commutes with the Fourier cosine transform.

\section{REFERENCES}

1. I. Schur, Bemerkung zur Theorie der beschränkten Bilinearformen mit unendlich vielen Veränderlichen, J. Reine Angew. Math. vol. 140 (1911) p. 23.

2. E. C. Titchmarsh, Introduction to the theory of Fourier integrals, Oxford, 1937, p. 93.

NORTHWESTERN UNIVERSITY 